\title{
Worse Renal Presentation and Prognosis in Initial-Onset Lupus Nephritis than Early-Onset Lupus Nephritis
}

\author{
Oh Chan Kwon, Jung Hwan Park, Sang-Won Lee, Jason Jungsik Song, Yong-Beom Park, and Min-Chan Park \\ Division of Rheumatology, Department of Internal Medicine, Yonsei University College of Medicine, Seoul, Korea.
}

\begin{abstract}
Purpose: To compare the clinical characteristics and renal outcomes between patients who initially had lupus nephritis (LN) at the onset of systemic lupus erythematosus (SLE) (initial-onset LN) and those who developed LN within 5 years after SLE onset (early-onset LN).

Materials and Methods: SLE patients with biopsy-proven LN were retrospectively reviewed. The clinical parameters and renal outcomes were compared between initial-onset and early-onset LN groups. We used Cox regression analysis to estimate risk of worse renal outcomes according to the onset time of $\mathrm{LN}$.

Results: Of all $136 \mathrm{LN}$ patients, 92 (67.6\%) and 44 (32.4\%) patients were classified into the initial-onset and early-onset LN groups, respectively. The initial-onset LN group had higher prevalences of class IV LN ( $54.3 \%$ vs. $34.1 \%, p=0.027)$, impaired renal function ( $34.8 \%$ vs. $11.4 \%, p=0.004)$, microscopic hematuria ( $73.9 \%$ vs. $54.5 \%, p=0.024)$, and higher urine protein/creatinine ratio [4626.1 (2180.0-6788.3) $\mathrm{mg} / \mathrm{g}$ vs. 2410.0 (1265.0-5168.5) $\mathrm{mg} / \mathrm{g}, p=0.006]$ at LN diagnosis. Renal relapse (46.3\% vs. $25.7 \%, p=0.039)$ and progression to chronic kidney disease (CKD) or end-stage renal disease (ESRD) were more common $(24.4 \%$ vs. $8.3 \%, p=0.042)$ in the initial-onset LN group. In Cox regression analysis, the initial-onset LN group had higher risks of renal relapse [adjusted hazard ratio (HR) 3.56, 95\% confidence interval (CI) 1.51-8.35, $p=0.004$ ] and progression to CKD or ESRD (adjusted HR 4.57, 95\% CI 1.03-20.17, $p=0.045$ ), compared with the early-onset LN group.

Conclusion: Patients with LN at SLE onset may have more severe renal presentations and experience worse renal outcomes than those who develop LN within 5 years.
\end{abstract}

Key Words: Systemic lupus erythematosus, lupus nephritis, prognosis

\section{INTRODUCTION}

Systemic lupus erythematosus (SLE) is an autoimmune disease that can have various multisystem manifestations. ${ }^{1}$ Among the numerous manifestations of SLE is lupus nephritis (LN).

Received: June 25, 2020 Revised: September 18, 2020

Accepted: September 21, 2020

Corresponding author: Min-Chan Park, MD, PhD, Division of Rheumatology, Department of Internal Medicine, Gangnam Severance Hospital, Yonsei University College of Medicine, 211 Eonju-ro, Gangnam-gu, Seoul 06273, Korea. Tel: 82-2-2019-3337, Fax: 82-2-2019-3508, E-mail: mcpark@yuhs.ac

-The authors have no potential conflicts of interest to disclose.

(C) Copyright: Yonsei University College of Medicine 2020

This is an Open Access article distributed under the terms of the Creative Commons Attribution Non-Commercial License (https://creativecommons.org/licenses/ by-nc/4.0) which permits unrestricted non-commercial use, distribution, and reproduction in any medium, provided the original work is properly cited.
Occurring in 40-75\% of patients with SLE, $\mathrm{LN}$ is associated with a poor prognosis in these patients. ${ }^{2} \mathrm{LN}$ may present with proteinuria, hematuria, active urinary sediment, or decreased renal function, ${ }^{3}$ and can progress to end-stage renal disease (ESRD). ${ }^{4}$ Although LN can develop at various time points during the disease course of SLE, it usually develops within 5 years of SLE onset $^{5,6}$ and more commonly at the onset of SLE (in approximately $50 \%$ of cases). ${ }^{7}$

The course and outcomes of LN are determined by an interplay of demographic, histopathological, and laboratory factors. ${ }^{8}$ Meanwhile, several studies have investigated whether the onset time of LN can affect the clinical presentations and outcomes of LN patients. ${ }^{9,10}$ Varela, et al. ${ }^{9}$ showed that the renal presentations and histopathologic patterns did not differ between early-onset LN (LN that developed within $\leq 5$ years after SLE diagnosis) and delayed LN (LN that developed $\geq 5$ years after SLE 
diagnosis). Another study reported worse renal outcomes in delayed-onset LN (mean interval between SLE diagnosis and LN diagnosis: 8.9 years) than in initial-onset LN (LN diagnosed at the onset of SLE).$^{10}$ However, to date, data are lacking on whether the clinical characteristics of renal disease differ between initial-onset LN and early-onset LN, which accounts for the majority of LN cases. Here, we aimed to compare clinical characteristics and renal outcomes between initial-onset LN and early-onset LN.

\section{MATERIALS AND METHODS}

\section{Study population}

Patients in whom LN was diagnosed using renal biopsy between July 2006 and December 2018 at two tertiary referral hospitals in Seoul, Korea were retrospectively selected for inclusion into this study. As our aim was to compare initial-onset LN and early-onset LN, which was not previously studied, patients who developed LN after $>5$ years of SLE diagnosis were excluded. All patients met the 1997 American College of Rheumatology classification criteria for SLE. ${ }^{11}$ For comparison, patients were categorized into two groups according to the onset time of LN: those who initially developed LN at the time of SLE onset (initialonset LN group) and those who developed LN within 5 years after SLE onset (early-onset LN group). Patients who had suspicious findings of nephritis (elevated creatinine, proteinuria, hematuria, pyuria, and urinary cast) at the time of SLE diagnosis and who underwent renal biopsy accordingly were categorized as initial-onset LN. Patients who did not have suspicious findings of nephritis and did not undergo renal biopsy at the time of SLE diagnosis, but developed suspicious findings of nephritis during follow up were categorized as early-onset LN. The patients in the early-onset LN group underwent renal biopsy at the time when suspicious findings of nephritis developed during follow up.

This study was approved by the Institutional Review Board of Gangnam Severance Hospital (IRB No: 3-2019-0072). Owing to the retrospective nature of this study, the requirement for informed consent was waived.

\section{Clinical parameters and medications}

We reviewed the following information recorded at the diagnosis of LN: age, sex, time from diagnosis of SLE to diagnosis of LN, presence of hypertension and diabetes mellitus, serum albumin and creatinine levels, estimated glomerular filtration rate (eGFR), antibodies to extractable nuclear antigens, lupus anticoagulant, anti- $\beta_{2}$ glycoprotein I antibody, anti-cardiolipin antibody, C3, C4, urinalysis results, urine protein/creatinine ratio (UPCR), presence of SLE manifestations other than renal disease, SLE Disease Activity Index (SLEDAI), ${ }^{12}$ International Society of Nephrology/Renal Pathology Society (ISN/RPS) 2003 classification, ${ }^{13}$ activity index, chronicity index, tubulointersti- tial inflammation, tubular atrophy, interstitial fibrosis, and glomerulosclerosis. For analysis, tubulointerstitial inflammation, tubular atrophy, and interstitial fibrosis were categorized into dichotomous variables (none-to-mild: $<25 \%$ of the acreage of interstitium affected vs. moderate-to-severe: $>25 \%$ of the acreage of interstitium affected). ${ }^{14}$ The severity of glomerulosclerosis was assessed as the proportion of sclerotic glomeruli to total glomeruli.

Medications prescribed during the observation period were also reviewed. Patients with proliferative LN (class III and class IV) and membranous LN (class V) with nephrotic range proteinuria received immunosuppressants. For induction therapy, patients received mycophenolate mofetil (2-3 g/day $)^{15}$ or cyclophosphamide according to the Euro-Lupus Nephritis regimen ${ }^{16}$ or the National Institutes of Health protocol ${ }^{17}$ with moderate-to-high dose glucocorticoid. After 4 weeks, glucocorticoid dosages were tapered according to the treating physicians' decision. Patients who failed to respond were switched from mycophenolate mofetil to cyclophosphamide (and vice versa) or received rituximab. For maintenance therapy, mycophenolate mofetil, azathioprine, or tacrolimus with low-dose glucocorticoid ( $\leq 7.5 \mathrm{mg}$ prednisolone or its equivalent) was used.

\section{Renal outcome}

For the comparison of renal outcomes between the two groups, achievement of a complete renal response (UPCR $<500 \mathrm{mg} / \mathrm{g}$ and normal GFR or within $10 \%$ of normal GFR if previously abnormal $)^{18}$ at 6 months after the initiation of induction therapy, occurrence of relapse, and progression to chronic kidney disease (CKD) or ESRD were evaluated. Relapse was defined as a $>25 \%$ decline in GFR, a $50 \%$ or more increase in proteinuria, or active urine sediment characterized by $>5$ red blood cell/highpower field and/or cellular casts. ${ }^{19} \mathrm{CKD}$ was defined as GFR $<60 \mathrm{~mL} / \mathrm{min} / 1.73 \mathrm{~m}^{2}$ for $\geq 3$ months, ${ }^{20}$ and ESRD was defined as requiring renal replacement therapy with $\mathrm{GFR}<15 \mathrm{~mL} /$ $\min / 1.73 \mathrm{~m}^{2}{ }^{20}$ The patients were retrospectively reviewed until they developed CKD or ESRD or until the last hospital visit, whichever occurred first.

\section{Statistical analysis}

Data are presented as means ( \pm standard deviations) or medians (interquartile range) for normal distributing or non-normal distributing continuous variables, respectively, and numbers (\%) for categorical variables. For the comparisons between groups, Student's t-test or the Mann-Whitney test was used for continuous variables, and Fisher's exact test (or chi-square test when appropriate) was used for categorical variables. The relapsefree survival rates and CKD- or ESRD-free survival rates were analyzed using Kaplan-Meier analysis and were compared using a log-rank test. We used Cox regression to estimate hazard ratios (HRs) for renal relapse and progression to CKD or ESRD, with the onset time of LN as the variable of interest. We initially performed an univariable analysis, and then performed mul- 
tivariable analyses adjusted for potential confounders. Multivariable models first included renal histologic parameters (ISN/ RPS class, activity index, and chronicity index) (model 1), followed by additional adjustment for other renal parameters (GFR, UPCR, and hematuria) (model 2), and use of hydroxychloroquine (HCQ) and immunosuppressive agents (model 3 ). A value of $p<0.05$ was considered statistically significant. All analyses were conducted using SPSS software (SPSS version 25.0; IBM Corporation, Armonk, NY, USA).

\section{RESULTS}

\section{Characteristics at the time of LN diagnosis}

A total of 179 patients with biopsy-confirmed LN, who met the American College of Rheumatology classification criteria for SLE, ${ }^{11}$ were identified. Forty-three patients in whom LN developed at $>5$ years after the diagnosis of SLE were excluded, and the remaining 136 patients were included for analysis. Of these 136 patients, $92(67.6 \%)$ patients were categorized as the initialonset LN group, and the other 44 (32.4\%) patients were categorized as the early-onset LN group. Patients in the early-onset LN group developed LN at a median of 25.1 (10.8-38.4) months after the diagnosis of SLE. The median observation period was 59.5 (21.3-94.3) months from the diagnosis of LN [Initial-onset LN group: 43.7 (15.5-88.8) months, and early-onset LN group: 80.7 (45.4-110.1) months]. A comparison of clinical characteristics at diagnosis of $\mathrm{LN}$ between the initial-onset and early-onset LN groups is shown in Table 1. Age ( $p=0.714)$, sex distribution ( $p=0.226)$, and the proportion of patients with hypertension ( $p=0.101)$ and diabetes mellitus ( $p=0.437)$ did not differ between groups. There were no significant differences in autoantibody profile between the two groups. C3 level [35.1 (24.6-56.8) mg/ dL vs. 52.7 (41.4-75.4) mg/dL, $p<0.001]$, C4 level [5.2 (2.6-9.3) $\mathrm{mg} / \mathrm{dL}$ vs. $11.0(5.2-18.0) \mathrm{mg} / \mathrm{dL}, p=0.001]$ and serum albumin level [2.6 (2.2-3.2) g/dL vs. $3.3(2.5-3.6) \mathrm{g} / \mathrm{dL}, p=0.005]$ were lower in the initial-onset LN group. In terms of SLE manifestations other than LN, serositis [20 (21.7\%) vs. 1 (2.3\%), $p=0.003$ ] and hematologic manifestations [49 (53.3\%) vs. 12 (27.3\%), $p=0.004]$ were more common in the initial-onset LN group. Further, SLEDAI ( $17.6 \pm 5.8$ vs. $14.5 \pm 6.2, p=0.005)$ was significantly higher in the initial-onset LN group. In comparison of renal parameters, patients in the initial-onset $\mathrm{LN}$ group more commonly had eGFR $<60 \mathrm{~mL} / \mathrm{min} / 1.73 \mathrm{~m}^{2}$ [32 (34.8\%) vs. 5 (11.4\%), $p=0.004]$, had higher UPCR [4626.1 (2180.0-6788.3) mg/g vs. 2140.0 (1265.0-5168.5) mg/g, $p=0.006]$, and more commonly had microscopic hematuria [68 (73.9\%) vs. 24 (54.5\%), $p=0.024$ ]. There were no significant differences in histologic data between the two groups, except the higher prevalence of class IV $\mathrm{LN}(54.3 \%$ vs. $34.1 \%, p=0.027)$ and lower chronicity index values $[1.0(0.0-2.0)$ vs. $2.0(1.0-3.0), p=0.028]$ in the initial-onset LN group.

\section{Comparison of treatment and renal outcomes according to the onset of $\mathrm{LN}$}

Patients who did not receive immunosuppressive therapy for LN (3 patients with class I LN, 4 patients with class II LN, and 7 patients with class V LN) were excluded from this analysis because the treatment and renal outcomes in these patients could not be determined. Eighty-six and 36 patients remained in the initial-onset LN and early-onset LN groups, respectively. In the induction phase, the use of HCQ ( $p=0.091)$, mycophenolate mofetil ( $p=0.327$ ), cyclophosphamide ( $p=0.266)$, and rituximab ( $p>0.999)$ did not differ between the two groups. However, patients in the initial-onset LN group received higher cumulative doses of glucocorticoid during the first 6 months (6548.4 $\pm 2826.8 \mathrm{mg}$ vs. $5098.4 \pm 2649.8 \mathrm{mg}, p=0.010$ ). After 6 months of induction treatment, a complete renal response was less commonly achieved in the initial-onset LN group [ 45 (52.3\%) vs. 26 (72.2\%), $p=0.042]$.

For the comparison of the medications used during the maintenance phase, patients who were refractory to induction therapy (6 patients in the initial-onset LN group and 1 patient in the early-onset LN group) were excluded, as the maintenance phase could not be determined in these patients. No significant differences were observed in the medications used (HCQ, $p=$ 0.070 ; mycophenolate mofetil, $p>0.999$; azathioprine, $p=0.227$; tacrolimus, $p=0.765$ ) in the maintenance phase. The two groups did not differ in regards to the use of angiotensin converting enzyme inhibitor or angiotensin II receptor blocker [71 (82.6\%) vs. 32 (88.9\%), $p=0.379]$.

The occurrence of relapse after remission was more common in the initial-onset LN group [37 (46.3\%) vs. 9 (25.7\%), $p=$ 0.039]. Moreover, the incidence of progression to CKD or ESRD was higher [21 (24.4\%) vs. $3(8.3 \%), p=0.042]$ in the initial-onset LN group (Table 2). Kaplan-Meier analysis of the two groups revealed significantly lower relapse-free survival $(p=0.005)$ (Fig. 1) and CKD- or ESRD-free survival ( $p=0.023)$ (Fig. 2) in the initial-onset LN group.

\section{Onset time of LN and risk of renal relapse and progression to CKD or ESRD}

Table 3 shows the risk of renal relapse and progression to CKD or ESRD in the initial-onset LN group, compared with the earlyonset LN group. In univariable analysis, initial-onset LN posed a higher risk of renal relapse [unadjusted HR 2.73, 95\% confidence interval (CI) 1.32-5.69, $p=0.007]$ and progression to CKD/ ESRD (unadjusted HR 4.20, 95\% CI 1.25-14.13, $p=0.020$ ). After adjusting for renal histologic parameters (multivariable analysis model 1), initial-onset $\mathrm{LN}$ was associated with higher risks of renal relapse (adjusted HR 3.47, 95\% CI 1.56-7.71, $p=0.002$ ) and progression to CKD/ESRD (adjusted HR 7.54, 95\% CI 1.9429.38, $p=0.004$ ). With additional adjustment for other renal parameters (multivariable analysis model 2), initial-onset LN was still significantly associated with higher risks of renal relapse (adjusted HR 3.12, 95\% CI 1.40-6.96, $p=0.006$ ) and pro- 
Table 1. Comparison of Baseline Characteristics According to the Onset of LN

\begin{tabular}{|c|c|c|c|}
\hline & Initial-onset LN (n=92) & Early-onset LN ( $n=44)$ & $p$ value \\
\hline Age (yr) & $35.7( \pm 16.5)$ & $36.8( \pm 13.8)$ & 0.714 \\
\hline Female sex & $80(87.0)$ & 42 (95.5) & 0.226 \\
\hline Hypertension & $24(26.1)$ & $6(13.6)$ & 0.101 \\
\hline Diabetes mellitus & $7(7.6)$ & $1(2.3)$ & 0.437 \\
\hline \multicolumn{4}{|l|}{ Serology } \\
\hline Anti-Sm Ab & $37(40.2)$ & $22(50.0)$ & 0.282 \\
\hline Anti-Ro Ab & $54(58.7)$ & $30(68.2)$ & 0.287 \\
\hline Anti-La Ab & $21(22.8)$ & $15(34.1)$ & 0.164 \\
\hline Anti-U1RNP Ab & $50(54.3)$ & $26(59.1)$ & 0.602 \\
\hline Anti-dsDNA Ab, median (IOR), IU/mL & $160.0(19.3-379.0)$ & $240.7(4.8-380.0)$ & 0.786 \\
\hline Lupus anticoagulant & $16(17.4)$ & $8(18.2)$ & 0.910 \\
\hline Anti- $\beta_{2}$ glycoprotein I Ab & $14(15.2)$ & $5(11.4)$ & 0.544 \\
\hline Anti-cardiolipin Ab & $24(26.1)$ & $13(29.5)$ & 0.672 \\
\hline C3, median (IQR), mg/dL & $35.1(24.6-56.8)$ & $52.7(41.4-75.4)$ & $<0.001$ \\
\hline C4, median (IOR), mg/dL & $5.2(2.6-9.3)$ & $11.0(5.2-18.0)$ & 0.001 \\
\hline Serum albumin, median (IOR), g/dL & $2.6(2.2-3.2)$ & $3.3(2.5-3.6)$ & 0.005 \\
\hline \multicolumn{4}{|l|}{ Other SLE manifestations } \\
\hline Mucocutaneous & $28(30.4)$ & $7(15.9)$ & 0.070 \\
\hline Musculoskeletal & $28(30.4)$ & $13(29.5)$ & 0.916 \\
\hline Neuropsychiatric & $7(7.6)$ & $3(6.8)$ & $>0.999$ \\
\hline Serositis & $20(21.7)$ & $1(2.3)$ & 0.003 \\
\hline Hematologic & 49 (53.3) & $12(27.3)$ & 0.004 \\
\hline SLEDAI & $17.6( \pm 5.8)$ & $14.5( \pm 6.2)$ & 0.005 \\
\hline \multicolumn{4}{|l|}{ Renal parameters } \\
\hline Serum Cr, median (IQR), mg/dL & $0.90(0.65-1.33)$ & $0.78(0.64-1.00)$ & 0.113 \\
\hline eGFR, median (IQR), mL/min/1.73 m² & $84.0(50.0-119.0)$ & $100.0(74.3-116.8)$ & 0.119 \\
\hline $\mathrm{eGFR}<60 \mathrm{~mL} / \mathrm{min} / 1.73 \mathrm{~m}^{2}$ & $32(34.8)$ & $5(11.4)$ & 0.004 \\
\hline Urine PCR, median (IOR), mg/g & $4626.1(2180.0-6788.3)$ & $2410.0(1265.0-5168.5)$ & 0.006 \\
\hline Urine $\mathrm{RBC} \geq 5 / \mathrm{HPF}$ & $68(73.9)$ & $24(54.5)$ & 0.024 \\
\hline Urine $\mathrm{WBC} \geq 5 / \mathrm{HPF}$ & $52(56.5)$ & $22(50.0)$ & 0.475 \\
\hline Urine cast & $18(19.6)$ & $5(11.4)$ & 0.233 \\
\hline \multicolumn{4}{|l|}{ ISN/RPS class } \\
\hline I & $2(2.2)$ & $1(2.3)$ & $>0.999$ \\
\hline$\|$ & $2(2.2)$ & $2(4.5)$ & 0.595 \\
\hline III & $18(19.6)$ & $12(27.3)$ & 0.311 \\
\hline$\| I+\mathrm{V}$ & $6(6.5)$ & $4(9.1)$ & 0.727 \\
\hline IV & $50(54.3)$ & $15(34.1)$ & 0.027 \\
\hline $\mathrm{IV}+\mathrm{V}$ & $6(6.5)$ & $3(6.8)$ & $>0.999$ \\
\hline V & $8(8.7)$ & $7(15.9)$ & 0.246 \\
\hline Activity index, mean median (IQR) & $7.5(4.0-10.3)$ & $6.0(1.0-10.0)$ & 0.404 \\
\hline Chronicity index, median (IOR) & $1.0(0.0-2.0)$ & $2.0(1.0-3.0)$ & 0.028 \\
\hline Moderate-to-severe TII & $14(15.2)$ & $4(9.1)$ & 0.324 \\
\hline Moderate-to-severe TA/IF & $3(3.3)$ & $3(6.8)$ & 0.388 \\
\hline Glomerulosclerosis, median (IQR) & $4.6(0.0-14.9)$ & $9.1(0.0-28.6)$ & 0.180 \\
\hline
\end{tabular}

LN, lupus nephritis; Ab, antibody; anti-dsDNA, anti-double-stranded DNA; SLE, systemic lupus erythematosus; SLEDAI, Systemic Lupus Erythematosus Disease Activity Index; $\mathrm{Cr}$, creatinine; eGFR, estimated glomerular filtration rate; PCR, protein/creatinine ratio; RBC, red blood cell; HPF, high-power field; WBC, white blood cell; ISN/RPS, International Society of Nephrology/Renal Pathology Society; TII, tubulointerstitial inflammation; TA, tubular atrophy; IF, interstitial fibrosis; SD, standard deviation; IQR, interquartile range.

Data are presented as mean \pm standard deviation or $\mathrm{n}(\%)$. 
Oh Chan Kwon, et al.

Table 2. Comparison of Treatment and Renal Outcomes According to the Onset of LN

\begin{tabular}{|c|c|c|c|}
\hline & Initial-onset LN ( $n=86$ ) & Early-onset LN ( $n=36)$ & $p$ value \\
\hline \multicolumn{4}{|l|}{ Medication in the induction phase } \\
\hline $\mathrm{HCO}$ & $56(65.1)$ & $29(80.6)$ & 0.091 \\
\hline MMF & $62(72.1)$ & $29(80.6)$ & 0.327 \\
\hline CYC & 38 (44.2) & $12(33.3)$ & 0.266 \\
\hline RTX & $2(2.3)$ & $0(0.0)$ & $>0.999$ \\
\hline Glucocorticoid, mg* & $6548.4( \pm 2826.8)$ & $5098.4( \pm 2649.8)$ & 0.010 \\
\hline Duration of induction phase, months & $8.1( \pm 3.3)$ & $7.8( \pm 3.9)$ & 0.595 \\
\hline Complete renal response at 6 months & $45(52.3)$ & 26 (72.2) & 0.042 \\
\hline Refractory to induction therapy & $6(7.0)$ & $1(2.8)$ & 0.672 \\
\hline \multicolumn{4}{|l|}{ Medication in the maintenance phase ${ }^{\dagger}$} \\
\hline $\mathrm{HCO}$ & $53(66.3)$ & $29(82.9)$ & 0.070 \\
\hline MMF & $74(92.5)$ & $32(91.4)$ & $>0.999$ \\
\hline AZA & $8(10.0)$ & $7(20.0)$ & 0.227 \\
\hline TAC & $18(22.5)$ & $7(20.0)$ & 0.765 \\
\hline ACE inhibitor or ARB & $71(82.6)$ & $32(88.9)$ & 0.379 \\
\hline Relapse after remission ${ }^{\dagger}$ & $37(46.3)$ & $9(25.7)$ & 0.039 \\
\hline Time from remission to relapse, median (IQR), months & $20.2(9.1-52.5)$ & $41.3(10.7-77.4)$ & 0.354 \\
\hline Progression to CKD or ESRD & $21(24.4)$ & $3(8.3)$ & 0.042 \\
\hline Time from LN to CKD or ESRD, median (IOR), months & $11.3(4.7-34.5)$ & $29.8(17.8-74.6)$ & 0.315 \\
\hline
\end{tabular}

LN, lupus nephritis; HCQ, hydroxychloroquine; MMF, mycophenolate mofetil; CYC, cyclophosphamide; RTX, rituximab; AZA, azathioprine; TAC, tacrolimus; ACE, angiotensin converting enzyme; ARB, angiotensin II receptor blocker; CKD, chronic kidney disease; ESRD, end-stage renal disease; SD, standard deviation; IQR, interquartile range.

Data are presented as mean \pm standard deviation or $\mathrm{n}(\%)$.

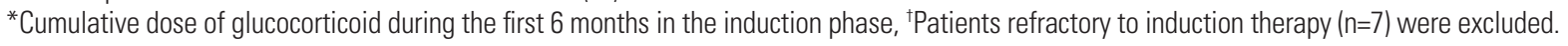

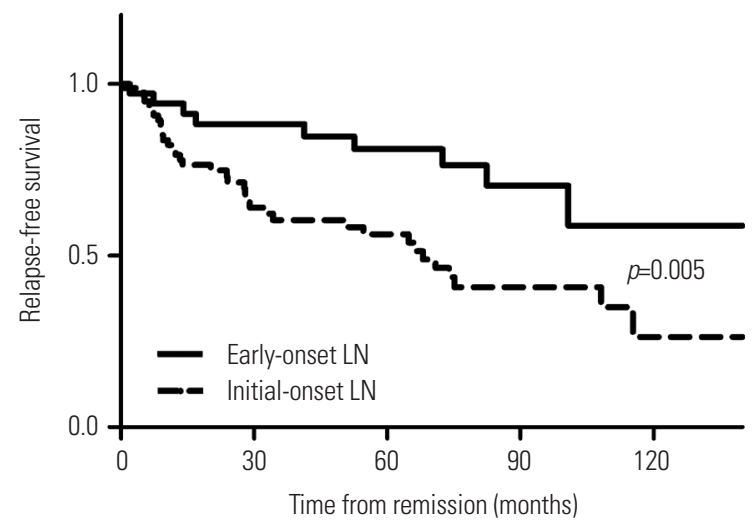

Number at risk

Early-onset LN

Initial-onset LN
35

80

$$
29
$$

37

Fig. 1. Kaplan-Meier survival curves for relapse-free survival. LN, lupus nephritis.

gression to CKD/ESRD (adjusted HR 4.39, 95\% CI 1.07-17.96, $p=0.040$ ). We obtained similar results after further adjustment for use of HCQ and immunosuppressive agents (multivariable analysis model 3).

\section{DISCUSSION}

In this study, we found that patients who initially developed LN

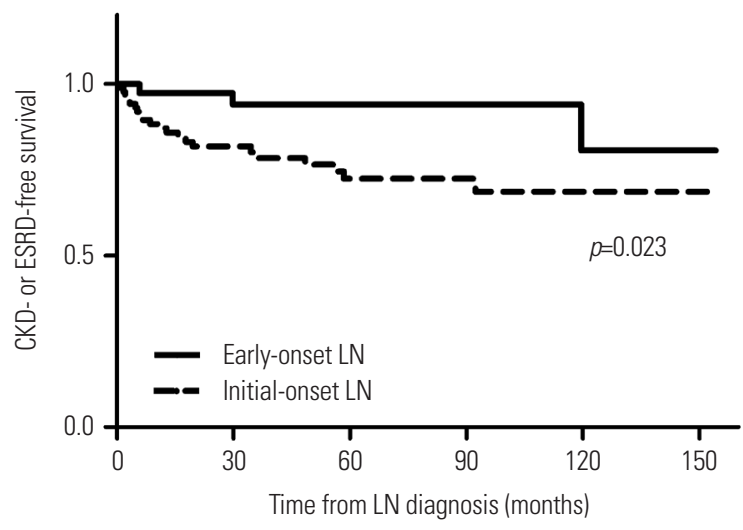

Number at risk

Early-onset LN Initial-onset LN

36

$$
30
$$$$
27
$$$$
16
$$$$
\begin{aligned}
& 16 \\
& 21
\end{aligned}
$$

Fig. 2. Kaplan-Meier survival curves for CKD- or ESRD-free survival. LN, lupus nephritis; CKD, chronic kidney disease; ESRD, end-stage renal disease.

at the time of SLE onset had more severe renal manifestations and faced a higher risk of worse renal outcomes than those who developed LN within 5 years after the diagnosis of SLE. To our knowledge, this is the first study to show differences in renal presentation and renal outcomes between initial-onset LN and early-onset LN.

A previous study reported no differences in pattern of glomerular lesions between early-onset LN and delayed $\mathrm{LN},{ }^{9}$ whereas a recent study reported a higher prevalence of class IV LN in 
Table 3. HRs for Renal Relapse and Progression to CKD/ESRD According to Onset Time of LN

\begin{tabular}{|c|c|c|c|c|c|c|c|c|}
\hline & \multicolumn{2}{|c|}{ Univariable analysis } & \multicolumn{2}{|c|}{$\begin{array}{l}\text { Multivariable analysis } \\
\text { model } 1^{*}\end{array}$} & \multicolumn{2}{|c|}{$\begin{array}{c}\text { Multivariable analysis } \\
\text { model } 2^{\dagger}\end{array}$} & \multicolumn{2}{|c|}{$\begin{array}{c}\text { Multivariable analysis } \\
\text { model } 3^{\ddagger}\end{array}$} \\
\hline & HR $(95 \%$ Cl) & $p$ value & HR $(95 \%$ Cl) & $p$ value & HR $(95 \%$ CI) & $p$ value & HR $(95 \%$ Cl) & $p$ value \\
\hline \multicolumn{9}{|l|}{ Renal relapse } \\
\hline Early-onset LN & 1.00 (reference) & & 1.00 (reference) & & 1.00 (reference) & & 1.00 (reference) & \\
\hline Initial-onset LN & $2.73(1.32-5.69)$ & 0.007 & $3.47(1.56-7.71)$ & 0.002 & $3.12(1.40-6.96)$ & 0.006 & $3.56(1.51-8.35)$ & 0.004 \\
\hline \multicolumn{9}{|c|}{ Progression to CKD/ESRD } \\
\hline Early-onset LN & 1.00 (reference) & & 1.00 (reference) & & 1.00 (reference) & & 1.00 (reference) & \\
\hline Initial-onset LN & $4.20(1.25-14.13)$ & 0.020 & 7.54 (1.94-29.38) & 0.004 & $4.39(1.07-17.96)$ & 0.040 & $4.57(1.03-20.17)$ & 0.045 \\
\hline
\end{tabular}

LN, lupus nephritis; CKD, chronic kidney disease; ESRD, end-stage renal disease; ISN/RPS, International Society of Nephrology/Renal Pathology Society (ISN/ RPS); GFR, glomerular filtration rate; UPCR, urine protein/creatinine ratio; $\mathrm{HCQ}$, hydroxychloroquine; HR, hazard ratio; $\mathrm{Cl}$, confidence interval.

${ }^{*}$ Adjusted for ISN/RPS class, activity index, chronicity index, ${ }^{\dagger}$ Adjusted for ISN/RPS class, activity index, chronicity index, GFR, UPCR, hematuria, ${ }^{\ddagger}$ Adjusted for ISN/RPS class, activity index, chronicity index, GFR, UPCR, hematuria, use of HCQ, and use of immunosuppressive agents.

early-onset LN than in delayed LN. ${ }^{21}$ Similar to this recent study, we observed differences in patterns of glomerular lesions between initial-onset $\mathrm{LN}$ and early-onset $\mathrm{LN}$ : the prevalence of class IV LN was higher in initial-onset LN than in early-onset LN. We also found that chronicity index values were significantly lower in the initial-onset LN group, although the difference in absolute values was only modest (median values of 1.0 and 2.0 for initial-onset LN group and early-onset LN group, respectively). The differences in histology, especially the higher prevalence of class IV LN, in the initial-onset LN might have contributed to the worse renal outcomes in the initial-onset LN group. However, in multivariable analysis model 1 , wherein we adjusted for renal histologic parameters, a higher risk of worse renal outcomes in the initial-onset LN group was observed. This suggests that the worse renal outcomes in the initial-onset LN group cannot be fully explained by differences in renal histology and that the disease course of LN may differ depending on the onset time of LN. Notably, achievement of a complete renal response at 6 months after starting induction treatment, which is predictive of good long-term renal outcome, ${ }^{22}$ was less common in the initial-onset $\mathrm{LN}$ group. This might have attributed to the worse renal outcomes in the initial-onset $\mathrm{LN}$ group.

C3, C4, and serum albumin levels were significantly lower, and other SLE manifestations, including serositis and hematologic manifestations, were more common in the initial-onset LN group, indicating higher systemic disease activity of SLE. Indeed, SLEDAI values were significantly higher in the initialonset LN group than in the early-onset LN group at the time of LN diagnosis. Considering that early-onset LN may develop in circumstances where systemic disease activity is relatively low, SLE patients without LN initially may benefit from careful renal monitoring even when systemic disease activity is low.

The initial-onset LN group more commonly had GFR $<60$ $\mathrm{mL} / \mathrm{min} / 1.73 \mathrm{~m}^{2}$ and hematuria, as well as higher amounts of proteinuria, at the time of LN diagnosis, suggesting more aggressive renal presentation in this groups. This might be a result of lead time bias. That is, as the patients in the early-onset LN group were being followed for their SLE, they were on rou- tine renal surveillance, which might have resulted in earlier detection and milder renal presentation. Furthermore, as the patients in the early-onset LN group were on treatment of SLE prior to the development of $\mathrm{LN}$, this might also have contributed to the milder renal presentation in this group. To minimize confounding from lead time bias, we adjusted for renal parameters in multivariable analysis model 2 , and the risks of renal relapse and progression to CKD or ESRD were higher in the initial-onset $\mathrm{LN}$ group. This suggests that the difference in renal outcomes between the initial-onset LN and early-onset LN groups was not likely the result of lead time bias. Furthermore, the association between initial-onset LN and worse renal outcome remained statistically significant when additionally adjusting for the use of HCQ and immunosuppressive agents, which are other potential confounders. Nevertheless, the potential mechanism underlying the difference in renal outcomes according to the onset time of $\mathrm{LN}$ is currently unclear and needs further investigation.

In a previous study comparing initial-onset LN and delayedonset LN (mean time from SLE to LN: 8.9 years), initial-onset LN had a better prognosis. ${ }^{10}$ More recently, another study comparing LN that developed within 1 year from SLE diagnosis (defined as early-LN in that study) and delayed-onset LN (mean time from SLE to LN: 8.9 years) showed that early LN had better outcomes. ${ }^{21}$ In our present study, the median time interval between SLE diagnosis and LN diagnosis in the early-onset LN group, which had more favorable renal outcomes than the initial-onset LN group, was 25.1 months (2.1 years). Taken together with the previous reports, ${ }^{10,21}$ we suspect that the renal prognosis of $\mathrm{LN}$ may differ depending on the onset time of $\mathrm{LN}$ diagnosis.

Our present study has several limitations. First, this was a retrospective study. Unidentified confounders may exist, which stems from the retrospective design. Second, although we included patients from two medical centers, all patients were Korean. Further studies with a larger number of patients from different ethnic populations may be helpful to confirm our results.

In summary, we showed that the renal characteristics and renal outcomes of LN may differ depending on its onset time. 
Patients who develop LN at the onset of SLE may have more severe renal presentations and may be at risk of worse renal outcomes than patients who develop LN within 5 years after SLE onset.

\section{ACKNOWLEDGEMENTS}

This study was supported by a faculty research grant from Yonsei University College of Medicine (6-2019-0111).

\section{AUTHOR CONTRIBUTIONS}

Conceptualization: Min-Chan Park. Data curation: all authors. Formal analysis: Oh Chan Kwon, Jung Hwan Park, and Min-Chan Park. Funding acquisition: Oh Chan Kwon. Investigation: Oh Chan Kwon and Min-Chan Park. Methodology: Oh Chan Kwon and Min-Chan Park. Project administration: all authors. Resources: all authors. Software: Oh Chan Kwon and Min-Chan Park. Supervision: Min-Chan Park. Validation: Oh Chan Kwon and Min-Chan Park. Visualization: Oh Chan Kwon and Min-Chan Park. Writing-original draft: Oh Chan Kwon and Min-Chan Park. Writing-review \& editing: Oh Chan Kwon and Min-Chan Park. Approval of final manuscript: all authors.

\section{ORCID iDs}

$\begin{array}{ll}\text { Oh Chan Kwon } & \text { https://orcid.org/0000-0001-7962-3697 } \\ \text { Jung Hwan Park } & \text { https://orcid.org/0000-0003-2911-2711 } \\ \text { Sang-Won Lee } & \text { https://orcid.org/0000-0002-8038-3341 } \\ \text { Jason Jungsik Song } & \text { https://orcid.org/0000-0003-0662-7704 } \\ \text { Yong-Beom Park } & \text { https://orcid.org/0000-0003-4695-8620 } \\ \text { Min-Chan Park } & \text { https://orcid.org/0000-0003-1189-7637 }\end{array}$

\section{REFERENCES}

1. Lisnevskaia L, Murphy G, Isenberg D. Systemic lupus erythematosus. Lancet 2014;384:1878-88.

2. Alba P, Bento L, Cuadrado MJ, Karim Y, Tungekar MF, Abbs I, et al. Anti-dsDNA, anti-Sm antibodies, and the lupus anticoagulant: significant factors associated with lupus nephritis. Ann Rheum Dis 2003;62:556-60.

3. Yu F, Haas M, Glassock R, Zhao MH. Redefining lupus nephritis: clinical implications of pathophysiologic subtypes. Nat Rev Nephrol 2017;13:483-95.

4. Croca SC, Rodrigues T, Isenberg DA. Assessment of a lupus nephritis cohort over a 30-year period. Rheumatology (Oxford) 2011; 50:1424-30.

5. Galindo-Izquierdo M, Rodriguez-Almaraz E, Pego-Reigosa JM, López-Longo FJ, Calvo-Alén J, Olivé A, et al. Characterization of Patients With Lupus Nephritis Included in a Large Cohort From the Spanish Society of Rheumatology Registry of Patients With Systemic Lupus Erythematosus (RELESSER). Medicine (Baltimore) 2016;95:e2891.

6. Imran TF, Yick F, Verma S, Estiverne C, Ogbonnaya-Odor C, Thiruvarudsothy S, et al. Lupus nephritis: an update. Clin Exp Nephrol 2016;20:1-13.

7. Anaya JM, Cañas C, Mantilla RD, Pineda-Tamayo R, Tobón GJ,
Herrera-Diaz C, et al. Lupus nephritis in Colombians: contrasts and comparisons with other populations. Clin Rev Allergy Immunol 2011;40:199-207.

8. Mok CC. Prognostic factors in lupus nephritis. Lupus 2005;14:3944.

9. Varela DC, Quintana G, Somers EC, Rojas-Villarraga A, Espinosa G, Hincapie ME, et al. Delayed lupus nephritis. Ann Rheum Dis 2008;67:1044-6.

10. Takahashi Y, Mizoue T, Suzuki A, Yamashita H, Kunimatsu J, Itoh $\mathrm{K}$, et al. Time of initial appearance of renal symptoms in the course of systemic lupus erythematosus as a prognostic factor for lupus nephritis. Mod Rheumatol 2009;19:293-301.

11. Hochberg MC. Updating the american college of rheumatology revised criteria for the classification of systemic lupus erythematosus. Arthritis Rheum 1997;40:1725.

12. Gladman DD, Ibañez D, Urowitz MB. Systemic lupus erythematosus disease activity index 2000. J Rheumatol 2002;29:288-91.

13. Weening JJ, D'Agati VD, Schwartz MM, Seshan SV, Alpers CE, Appel GB, et al. The classification of glomerulonephritis in systemic lupus erythematosus revisited. J Am Soc Nephrol 2004;15:241-50.

14. Yu F, Wu LH, Tan Y, Li LH, Wang CL, Wang WK, et al. Tubulointerstitial lesions of patients with lupus nephritis classified by the 2003 International Society of Nephrology and Renal Pathology Society system. Kidney Int 2010;77:820-9.

15. Chan TM, Li FK, Tang CS, Wong RW, Fang GX, Ji YL, et al. Efficacy of mycophenolate mofetil in patients with diffuse proliferative lupus nephritis. Hong Kong-Guangzhou Nephrology Study Group. N Engl J Med 2000;343:1156-62.

16. Houssiau FA, Vasconcelos C, D'Cruz D, Sebastiani GD, Garrido Ed Ede R, Danieli MG, et al. Immunosuppressive therapy in lupus nephritis: the Euro-Lupus Nephritis Trial, a randomized trial of low-dose versus high-dose intravenous cyclophosphamide. Arthritis Rheum 2002;46:2121-31.

17. Austin HA 3rd, Klippel JH, Balow JE, le Riche NG, Steinberg AD, Plotz $\mathrm{PH}$, et al. Therapy of lupus nephritis. Controlled trial of prednisone and cytotoxic drugs. N Engl J Med 1986;314:614-9.

18. Bertsias GK, Tektonidou M, Amoura Z, Aringer M, Bajema I, Berden $\mathrm{JH}$, et al. Joint European League Against Rheumatism and European Renal Association-European Dialysis and Transplant Association (EULAR/ERA-EDTA) recommendations for the management of adult and paediatric lupus nephritis. Ann Rheum Dis 2012; 71:1771-82.

19. Gibson KL, Gipson DS, Massengill SA, Dooley MA, Primack WA, Ferris MA, et al. Predictors of relapse and end stage kidney disease in proliferative lupus nephritis: focus on children, adolescents, and young adults. Clin J Am Soc Nephrol 2009;4:1962-7.

20. Inker LA, Astor BC, Fox CH, Isakova T, Lash JP, Peralta CA, et al. KDOQI US commentary on the 2012 KDIGO clinical practice guideline for the evaluation and management of CKD. Am J Kidney Dis 2014;63:713-35.

21. Nakano M, Kubo K, Shirota Y, Iwasaki Y, Takahashi Y, Igari T, et al Delayed lupus nephritis in the course of systemic lupus erythematosus is associated with a poorer treatment response: a multicentre, retrospective cohort study in Japan. Lupus 2019;28:1062-73.

22. Tamirou F, D'Cruz D, Sangle S, Remy P, Vasconcelos C, Fiehn C, et al. Long-term follow-up of the MAINTAIN Nephritis Trial, comparing azathioprine and mycophenolate mofetil as maintenance therapy of lupus nephritis. Ann Rheum Dis 2016;75:526-31. 